\title{
La antropología de la nueva normalidad: una realidad digital
}

\author{
TATIANA ALEXANDRA CRUZ \\ Antropolab-Redlab de Antropología aplicada | Bogotá, Colômbia \\ talexcruzana@gmail.com
}

DOI 10.11606/issn.2316-9133.v30i1pe185625

resumen En este artículo se abordará el campo de la antropología digital desde la comprensión de sus orígenes y de los personajes que han aportado a la construcción teórica y práctica de esta disciplina. Se explorarán los diversos campos donde el papel de la antropología digital es fundamental para explicar fenómenos sociales, culturales y tecnológicos. También se propondrá una reflexión sobre el cuestionamiento de la disciplina con miras al entendimiento de la aplicabilidad de la antropología digital en el campo laboral, tomando como ejemplo el caso colombiano y los hallazgos que al respecto arroja la "Encuesta sobre el mercado laboral de la antropología en Colombia" desarrollada por Antropolab.

palabras clave Antropología digital. Ciberantropología. Códigos digitales. Etnografía. Redes sociales.

The Anthropology of the new normality: a digital reality

abstract This article will address the field of digital anthropology. We will begin discerning its origins and the researchers who contributed to its theoretical and practical construction. We will explore the diverse fields where digital anthropology's role is essential to explain social, cultural, and technological phenomena. We will also propose a reflection about the demand made to the discipline to comprehend the applicability of digital anthropology in the labor market, taking as an example the Colombian case and the findings made by the "Survey about the anthropology labor market in Colombia" developed by antropolab.

keywords Digital anthropology. Ciberanthropology. Digital codes. ethnography. Social network.

A antropología da nova normalidade: uma realidade digital 
resumo Este artigo discorrerá sobre o campo da antropologia digital a partir da compreensão de suas origens e das personagens que tem contribuído à construção teórica e prática desta disciplina. Serão explorados os diversos campos onde o papel da antropologia digital é fundamental para explicar fenômenos sociais, culturais e tecnológicos. Também será proposta uma reflexão sobre o questionamento da disciplina para compreender o emprego da antropologia digital no campo laboral, tendo como exemplo o caso colombiano e as descobertas que sobre isso apresenta a "pesquisa sobre mercado de trabalho da antropologia na Colômbia" desenvolvido por Antropolab.

palavras-chave Antropologia digital. Ciberantropologia. Códigos digitais. Etnografia. Redes sociais.

\section{¿Qué es la Antropología digital?}

Llamamos antropología digital ${ }^{1}$ al estudio de la vida humana que se desarrolla en escenarios digitales. La antropología digital se ocupa de estudiar lo los diferentes aspectos que componen lo digital, como los espacios digitales (las redes sociales, apps, y videojuegos en línea), los códigos digitales (memes, gifs, emoticones), e indaga sobre temáticas individuales (identidad y diversidad) y temáticas colectivas (afiliación, comunidades, redes y nodos digitales). La antropología digital además se ocupa de entender y clarificar la relación humano-máquina bidireccionalmente generando visibilidad en los cambios tecnológicos dados por el hombre y los cambios que éste ha tenido a causa de la inserción de la tecnología en la vida cotidiana.

Al hablar de antropología digital es importante reconocer el trabajo de Daniel Miller (1998; 2011), Daniel Miller y Don Slater (2000), Sarah Pink (2009; 2012) y Pink, Horst y Postill (2019) con quienes han hecho aportes contundentes y relevantes a la conformación teórica y práctica de la antropología digital y los estudios digitales. Daniel Miller, profesor de antropología de la Universidad de Cambridge, comenzó su indagación entendiendo la relación materialista del ser humano y lo hizo explorando diversos elementos que hacen parte de su cotidianidad, como la ropa que usamos, los artículos de uso diario y el internet. Este pensamiento le permitió identificar el uso de tecnología en términos de impacto, transformación y, por qué no, hibridación humano/máquina. Entre los trabajos más relevantes del profesor Miller se encuentra un estudio del año 2000 sobre el efecto de Facebook en la vida cotidiana que construye el marco teórico para el estudio de redes sociales en las que ha seguido trabajando.

\footnotetext{
${ }^{1}$ Más referencias dirigirse a: https://www.antropolab-red.com/, https://digital-ethnography.com/team/danielmiller/, https://digital-ethnography.com/team/sarah-pink/ y https://www.triciawang.com/
} 
Al hablar del trabajo en redes sociales del profesor Miller, es fundamental mencionar el concepto de Polimedia. Daniel Miller describe la polimedia como la estructura de posibilidades por las cuales un Individuo gestiona sus relaciones emocionales y afectivas. La polimedia permite identificar qué cuestiones emocionales, culturales y morales entran en juego cuando los seres humanos interactúan en estos escenarios digitales. Del mismo modo que en la vida real hablamos de que un corpus cultural, un corpus regional y un corpus geográfico son factores que influyen en la relación de una persona con cualquier otra persona en la vida real-offline, estos mismos cuestionamientos morales y culturales se ponen en juego en la vida virtual-online.

Direccionado por estas ideas y entendiendo que el escenario digital requiere mayor investigación y conocimiento de lo que allí se produce, Miller y su equipo lograron en el 2009 la creación el de la maestría en antropología digital de la Universidad de College of LondonUCL que es hoy reconocida a nivel mundial como la mejor escuela de antropología digital. En el 2016 la imprenta de la UCL produjo la serie de libros Why we post con la que Miller y un grupo de antropólogos intentan dar respuesta a preguntas como ¿por qué publicamos en las redes sociales? O, ¿estamos reemplazando las relaciones cara a cara -face to face-por las relaciones en pantalla on-screen?

El trabajo de Miller como precursor de la antropología digital es ayudarnos a entender que el mundo offline (escenario real, no digital) no es el único donde se desarrolla la vida en comunidad; más aún, nos invita a pensar en que hay otro escenario: el online (escenario digital) donde también se desarrolla la vida cultural, social, política y económica que se desarrolla paralelo al real y en el cual las dinámicas que allí tiene lugar gozan de particularidades que deben ser objeto de estudio de la antropología.

Por su parte Sarah Pink, doctora en antropología de la Universidad de Kent en Inglaterra, investigadora y autora prolífica es la autoridad en etnografía digital y sensorial; ha trabajado no sólo generando teoría y metodología para la etnografía en escenarios digitales, si no que además ha hecho contribuciones valiosas al desarrollo de la antropología digital. También ha hecho importantes colaboraciones con el mundo del diseño, marketing y la industria llevando la etnografía digital a conversar con otras disciplinas. Entre los logros de la doctora Pink, está la introducción del concepto de materialidad digital en el que propone que una persona puede estar en el otro lado del mundo cuando se habla de offline, sin embargo, puede estar presente a través de una video llamada en ese mundo online, lo que en resumidas cuentas habla del paralelismo de la vida en off y en on. Este concepto permite reinterpretar, moldear y reestructurar la etnografía como instrumento de investigación y contextualizarla al mundo online como uno de los pilares de investigación que tenemos los antropólogos digitales.

Sarah Pink nos da el instrumento contextualizado al mundo on y abre el escenario para el trabajo colaborativo con diversas áreas del conocimiento, posicionando así la antropología digital como aliado estratégico de investigación y creación de conocimiento en entornos online. 
El entendimiento de los escenarios digitales y de la dinámica humana en estos escenarios nos permite identificar sucesos que han ocurrido desde que la tecnología digital entró a ser parte de la vida cotidiana de las personas, también genera una instantánea de lo que hoy en día es el mundo, cuáles son sus relaciones sociales, culturales, emocionales, y económicas entre otros; además, nos permite generar estudios predictivos que pueden darnos alertas tempranas de problemáticas que emerjan en estos escenarios online, y anticiparnos a cambios importantes en la vida social que conocemos, lo que nos lleva a pensar cuáles son las siguientes etapas de la realidad digital en términos económicos y culturales, por mencionar dos de los más importantes.

La antropología digital nace a partir de diferentes preguntas de la relación de los seres humanos con los diferentes escenarios digitales, surge a partir de la necesidad de Identificar y visibilizar las dinámicas que allí tenían lugar. La antropología digital da respuestas a preguntas como ¿cómo se genera identidad dentro de una red social? ¿Cómo se genera afiliación a un colectivo dentro del escenario digital si hablamos, por ejemplo, de comunidades redes y nodos? ¿Cuál es el tránsito de dinero que se puede dar en los escenarios digitales y cuáles son los parámetros con por los cuales se mueve dicho dinero? ¿Qué tipo de contenido publican los usuarios cuando ingresan a las redes sociales o a escenarios digitales? Éstas son sólo algunas de las preguntas que se pueden responder a través de la antropología digital.

Volvamos un momento a dos de los conceptos básicos de la antropología digital, el on y el off. Cuando hablamos de escenarios on y off nos referimos a los escenarios en los cuales se desarrolla la vida del ser humano. Vamos a hablar en primer lugar del escenario off que quiere decir fuera de línea, o sea, todos esos espacios donde se desarrolla la vida del ser humano y que no están condicionados por un escenario digital. Podríamos hablar de que el desarrollo de la vida en comunidad se dio primero en espacios comunales como las casas, los parques, y los centros de entretenimiento. Para algunas personas el offline se traduce como la vida real en oposición al online como vida virtual.

Por otro lado, el escenario online se refiere al entorno atravesado por la tecnología como una red social, una aplicación app, un videojuego en línea o cualquier otro espacio tecnológico que nos permita interactuar entre seres humanos o con una interface. Hoy en día los escenarios online no sólo sirven como espacios de desarrollo de la vida social sino también son el espacio que nos permite un desarrollo de una vida social, económica, política, amorosa, e intelectual, por nombrar algunos

Obviamente cada uno de estos espacios, el online y el offline, tienen particularidades que permiten el desarrollo de ciertas dinámicas humanas precisas. Por ejemplo, el desarrollo de la vida emocional en términos amorosos; podríamos decir que el espacio offline nos permite un encuentro cara a cara, una charla en persona, un reconocimiento del lenguaje corporal en directo, y también nos permite identificar cómo nos sentimos con relación a esa persona que tenemos enfrente, cuáles son esas emociones que experimentamos estando cerca a otro ser 
humano. La dificultad para muchas personas es llegar a ese nivel de cercanía con el otro, es decir, el problema está en encontrar a este otro con el que tener un encuentro cara a cara.

Ahora bien, si hablamos del escenario online que nos permite hacer un acercamiento en términos emocionales, amorosos, románticos o sexuales, podríamos identificar una aplicación como Tinder que nos permite relacionarnos con otros seres humanos en la misma búsqueda. La premisa es simple, la aplicación contiene una base de datos que le permite al usuario hacer la búsqueda de una pareja potencial basada en criterios específicos como edad, gustos en común y distancia física. El usuario tiene a su disposición una selección de otros usuarios con los que en teoría podría haber un encuentro. La dificultad está ahora en hacer una conexión a nivel real.

Otro ejemplo de la vida online versus la vida offline es la transmisión de un mensaje. En el pasado existía solamente el escenario offline cuando se necesitaba transmitir un mensaje, sólo se tenía la posibilidad de la comunicación persona a persona, es decir, comunicación offline uno a uno... muy útil siempre y cuando los dos interlocutores se encuentren en el mismo espacio físico y en el mismo tiempo. Con la expansión de las sociedades se hizo necesaria la transmisión de mensajes entre distancias significativas; la implementación de sistemas de mensajería como el correo postal y el telegrama permitieron la comunicación entre un emisor y un receptor solucionando el problema de la distancia física, pero no en tiempo real, seguimos estando offline.

La entrada del teléfono fijo y del telégrafo como solución al problema del espacio y el tiempo en la comunicación es el primer acercamiento online a la comunicación; el mensaje se transmite en directo sin importar la distancia física. Por supuesto la tecnología y el desarrollo no se han contentado con este acercamiento: hemos pasado del mensaje en persona a la carta y de la carta al correo electrónico; de la conversación en persona a la llamada, y de la llamada a la videollamada; del offline al online. Se gana en acortar distancias y en inmediatez: dos grandes cualidades que trae el mundo digital.

\section{¿Qué es lo digital en lo comportamental?}

Para responder esto necesitamos seguir poniendo en paralelo el off y el on, el off es análogo y el on es digital. Cuando hablamos de lo digital hablamos de dos componentes importantes: uno es la tecnología que se encuentra en nuestros celulares, computadoras, tabletas

y otros objetos digitales que usamos a diario; y el otro es el relacionamiento de la vida cotidiana con esta tecnología, lo comportamental. Es decir, cuando hablamos de lo comportamental en lo digital hablamos de un aspecto que no es palpable y sin embargo es medible, estamos hablando de algo que no podemos tocar, pero que sí podemos medir para identificar, enumerar, investigar y ver nuestro comportamiento a través de la pantalla, nuestro comportamiento frente a la revolución digital. 
El término "revolución digital" nos habla de una revolución cimentada en las nuevas tecnologías y en las tecnologías que están al servicio de las nuevas comunicaciones. Sin embargo, también llamamos revolución digital al momento en el que la tecnología empieza a ser parte de la vida cotidiana de los seres humanos, es decir, no sólo hablamos en términos de avances tecnológicos de sectores ultra desarrollados, sino que también hablamos de un cambio comportamental, un cambio de pensamiento y un cambio de paradigma en la vida de los seres humanos.

La revolución digital también tiene que ver con lo smart. Cuando nos referimos a este término hablamos de todas las funcionalidades que hacen un proceso mucho más inteligente o que acorta tiempos, fases y eslabones en la cadena de producción de cualquier tipo de actividad. Entonces hablamos de los smartphones, de las ciudades smart, de la tecnología smart que permite hablar de un mundo mucho más inteligente y funcional, y en el centro se encuentra el internet como posibilitador, como eje articulador de la vida cotidiana, de la tecnología y de lo digital.

Sumado a esto tenemos un cambio de mentalidad, un cambio de paradigma en los seres humanos que entendemos la tecnología no sólo como un invento, una máquina, o un proceso tecnológico, sino que percibimos ésta revolución digital como una nueva dirección en la vida humana donde la relación human-machine o ser humano y máquina se da en términos de corresponsabilidad y es bidireccional. En esa medida entendemos que las tecnologías son permeadas por los seres humanos y que los seres humanos se permean en su cotidianidad a través del uso de estas tecnologías y de este escenario digital.

Las realidades a las que hoy se enfrenta la humanidad están atravesadas por el escenario digital, sin ninguna duda. La revolución digital no sólo genera un cambio en términos de la vida cotidiana sino un cambio también en términos del paradigma de dónde, cómo, cuándo y por qué se generan las dinámicas socioculturales de los seres humanos. Al no estudiar las dinámicas que se tejen en estos escenarios digitales nos estaremos perdiendo partes del estudio de la vida humana; es decir, cuando queremos hablar de un estudio 360 grados, estamos hablando de un estudio en todos los aspectos que envuelven la vida de los seres humanos, y lo digital no es la excepción. Si no hemos entendido que el escenario digital hace parte del desarrollo de esa vida estaremos viendo sólo un fragmento que puede no dar respuestas totales a preguntas que surgen en términos de dinámicas socioculturales. Una analogía de esto es la luna y lo que vemos de ella. La luna tiene una parte visible y una parte oscura, si sólo nos quedáramos con el entendimiento de la investigación que se ha hecho en la parte luminosa de la luna, en realidad no podremos entender cuál es la dinámica de este satélite natural, sólo conoceremos una realidad fraccionada que no es representativa de un todo.

Lo mismo ocurre con la antropología digital y la vida humana. Hemos entendido, hemos investigado, hemos visibilizado qué es lo que pasa en la vida cotidiana del ser humano en el escenario offline, pero la realidad de las dinámicas del ser humano no pueden estudiarse, 
entenderse, e identificarse en su totalidad si no tenemos el referente de la tercera revolución; la digital; más aún, no podremos hacer predicciones sobre la cuarta revolución y a dónde nos conducirá, cuáles son los posibles avances, descubrimientos y aplicabilidades de estos entornos digitales como respuestas a problemáticas actuales y futuras.

Desde el desarrollo del transistor a principios de los 50, hasta el smartphone que conocemos en este momento, la revolución digital ha avanzado como ningún otro movimiento de desarrollo humano en términos de velocidad y cubrimiento. Pasamos de transistores a lenguajes de programación en el 1954, a circuitos integrados en el 58, luego al computador personal en el 71 y al 2021 donde somos capaces de hacer una video llamada en tiempo real a cualquier persona en el mundo que cuente con un dispositivo digital y con red; o sea, la mayoría. Estos setenta años de avance podrían parecer irrisorios para muchos, pero si consideramos que el primer registro escrito del ser humano data de hace más de cinco mil años, es casi irreal pensar en la cantidad de correos electrónicos que podemos enviar y las llamadas que podemos hacer en una hora. Por supuesto este cambio tan veloz y radical tiene consecuencias para el ser humano y por ende necesita un estudio profundo y riguroso: la antropología digital.

Del mismo modo en que las ciencias sociales, entre ellas la antropología, se han encargado de revisar y estudiar los movimientos humanos y sus desarrollos y revoluciones, esta nueva revolución debe ser revisada, estudiada y medida. Centrémonos en las revoluciones del desarrollo humano en su ser industrial; la primera trajo la mecanización del trabajo, la segunda el motor, la tercera las comunicaciones electrónicas y la cuarta, la que nos concierne más, la conexión digital a gran escala. Las primeras dos revoluciones industriales fueron análogas y las siguientes digitales. En la tercera revolución está el internet, la intercomunicación y las redes sociales. El concepto de lo smart es la antesala de un cuarto movimiento donde prima "el internet de las cosas", los dispositivos conectados y la robótica, las fábricas 4.0 enmarcadas en el Big Data, la Inteligencia artificial y el machine learning.

Ahora, ¿cómo nos relacionamos los seres humanos con lo digital? Para abordar esta pregunta tenemos que hablar de seres humanos que pertenecen a diferentes generaciones, entendiendo que cada una de ellas se relaciona de manera diferente con los entornos digitales. Para ejemplificar un poco podríamos proponer un parangón entre una persona nacida dentro de lo que llamamos generación $Z$, quien desde el principio de su vida hace uso del entorno digital, lo entiende como parte del diario vivir y sabe moverse dentro de diferentes escenarios, por lo que podríamos decir que es tridimensional en ese entendimiento de cómo funciona el espacio online y offline, Por otro lado, tenemos una generación un poco anterior, los llamados millennials, que han nacido en un mundo no digitalizado y han tenido que ver el nacimiento de lo digital, y a quienes su trabajo, aficiones, enlaces sociales les requieren una relación con el entorno digital, con la tecnología y la han vuelto parte de su día día; es decir, nacieron análogos pero son muy capaces de entender y procesar el entorno digital. Caso diferente es el de la 
generación de baby boomers que no dependieron del espacio digital para el desarrollo de su trabajo o de su vida social, pero que han aprendido a usar este entorno para relacionarse afectiva y socialmente, algunos con mucho más esfuerzo que otros.

En el trabajo y en la cotidianidad estas tres generaciones se desenvuelven de manera diferente; en general los baby boomers prefieren las facturas físicas, los pagos en ventanilla de banco y el teléfono para solicitar un taxi. Los millennials reciben correspondencia física y virtual, son capaces de realizar transacciones virtuales y en persona, y son capaces de solicitar una cita médica por teléfono o en una plataforma virtual. Los más jovencitos tienen el mundo digital a sus pies, son capaces de usar las redes disponibles para todos los servicios que necesitan y para su interacción con el otro, pero probablemente no se sienten cómodos si el único medio disponible para solicitar una información es hacer una llamada telefónica.

\section{Lo digital en el contexto actual}

Lo digital está en diferentes esferas de la vida cotidiana y es transversal a todos los espacios de la vida, no solo en lo social, sino también la vida económica, política, cultural, e incluso religiosa. Frente a la pregunta de por qué es importante enunciar esta realidad, la respuesta es sencilla, no somos aun completamente conscientes de lo digital en otro escenario fuera de las redes sociales.

Hablar de lo digital en términos económicos refiere a hablar por ejemplo de la idea de tener acceso a los servicios bancarios desde el celular, de la posibilidad de realizar transacciones que antes eran imposibles sin estar presente físicamente en las sucursales de los mismo; hablar de lo digital en el ámbito de la salud es hablar de una consulta virtual con un médico, una revisión offline que genera igualmente una prescripción de medicamentos e incluso una remisión al especialista. Hablar de lo digital en la educación es hablar de la posibilidad de asistir a una clase virtual en tiempo real y hasta de una clase asincrónica, que es una clase impartida antes de que el estudiante se siente frente a la pantalla a recibir el conocimiento. Hablar de lo digital es hablar de la posibilidad de asistir virtualmente a celebraciones religiosas, como una misa en semana santa, lo que es una idea revolucionaria si tenemos en cuenta que el Vaticano es una institución profundamente conservadora.

Obviamente hablar de lo digital refiere también a hablar de adelantos en ciencia, tecnología, e innovación qué son el referente más común que tenemos cuando de hablamos de lo digital, además de las redes sociales; sin embargo, abordar la cuestión del fenómeno de lo digital, es hablar de todos los ámbitos de nuestra vida actual, algo de lo que somos en mayor o menor medida conocedores, pero no totalmente conscientes.

En el año 2020 el Centro de Desarrollo de la Organización para la Cooperación y el Desarrollo Económico (OCDE), La Comisión Económica para América Latina y el Caribe (CEPAL) de las Naciones Unidas, el Banco de Desarrollo de América Latina (CAF) y la Comisión 
Europea socializaron el informe de perspectivas económicas de América Latina, que plantea la idea de que el camino a una recuperación después de la crisis actual del COVID-19 está en la transformación digital, donde el uso de lo digital para mejorar procesos no sólo en el sector privado, si no también en el sector público.

No obstante, el mismo documento resalta que "el acceso a Internet, en particular, está lejos de ser universal: en 2018, el 68\% de la población de ALC lo usó regularmente, casi el doble de la proporción en 2010, pero muy por debajo del promedio de la OCDE de $84 \%$. Además, mientras que el 75\% de la población más rica de América Latina usa Internet, solo el 37\% de la población más pobre lo hace. La diferencia entre ricos y pobres es mucho mayor (casi 40 puntos porcentuales) en ALC que en los países de la OCDE (menos de 25 puntos porcentuales).” Esto pone en evidencia un panorama heterogéneo y desigual en términos de acceso, uso y brechas de clase que necesitan ser estudiado, analizado, abordado y solucionado.

Los entornos de desigualdad, de injusticia, y pobreza no están exentos de ser planteados como temáticas en lo digital; por el contrario, las causas sociales han encontrado en este escenario digital un entorno con un público ávido de información, de expresión y de acción. Este uso debe ser analizado a la luz de las dos facetas que presenta. Por un lado, permite evidentemente la expresión de la inconformidad, de la desigualdad, y de propuestas para la construcción de entornos más dignos para las poblaciones. Esta apertura ha permitido darle voz a comunidades invisibilizadas, ha dado lugar a colectivos críticos y ha concentrado a diversos públicos en torno a acciones de movilización ciudadana. De igual manera, esta herramienta posibilita el tránsito de información relevante para todos los públicos, el acceso a discursos que de otro modo no podrían alcanzar a algunas poblaciones.

Esta es, por decirlo de algún modo, la cara amable de la red como entorno de movilización, pero no es su totalidad. También es posible usarla con fines oscuros, desinformativos, violentos y en ocasiones terriblemente perversos. Basta con preguntarnos cuántas veces hemos vistos cadenas de whatsapp con fake news, cuántas veces hemos visto a los haters amenazando de muerte a otros e infligiendo temor por su integridad física y mental, y cuántos hemos escuchado una historia de personas que fueron desaparecidas o asesinadas por una persona o personas con quienes mantenían una relación a través de la virtualidad. En este medio se vale todo y los jugadores de todos los bandos están listos para aprovechar la oportunidad de expresarse... muchas veces el mensaje es irrelevante, lo importante es generar ruido y visibilidad.

La irrupción de la pandemia actual en la cotidianidad de los seres humanos de todo el mundo es para el campo de lo digital no sólo una gran oportunidad de expansión, sino una herramienta fundamental para proponer alternativas, desarrollo y productos que permitan a la humanidad no sacrificar apartes de la vida cotidiana al aislamiento. El cambio que nos trajo la pandemia sobrepasó la problemática de lo netamente médico; el mundo entero se paralizó a 
causa de la COVID-19. Las medidas preventivas y correctivas incluyen el cese de actividades de centros donde se aglomeraba la población, el encierro casi completo de ciudades y países, y el cierre de fronteras entre otras medidas antes impensables, lo que ocasionó que las tareas comunes como hacer la compra, estudiar o trabajar tuvieran que ser repensadas y apoyadas por soluciones digitales. La vida social, familiar, laboral, escolar y física (en términos de actividad y ejercicio) se trasladó de múltiples escenarios a uno solo: el hogar, y en concreto, a las pantallas digitales.

La nueva normalidad es un término que explica la realidad que deja esta pandemia: declara que la realidad actual se cimienta en lo digital, es el centro de toda operación, comunicación y entretenimiento; es el soporte y el medio que nos permite mantenernos a flote en medio de la incertidumbre. La industria, el comercio, las instituciones educativas, los centros de entretenimiento se ven en la necesidad de transformarse en centros digitales obligatoriamente si quieren seguir siendo relevantes y no caer en la obsolescencia. El marco de esta pandemia ha ayudado a acelerar el desarrollo digital pues en gran medida recurrimos a esta posibilidad para dar soluciones reales a las problemáticas que la crisis mundial nos ha traído, confiamos en la capacidad del mundo digital para continuar con nuestras rutinas y al mismo tiempo le exigimos que cada vez sea más performing.

\section{Antropología digital: campos de acción y academia}

En esta nueva normalidad la presencia digital está al servicio de todos. Cuando hablamos de la presencia de lo digital en empresas, gobiernos, comunidades educativas, y otros grupos económicos y sociales influyentes, es claro que estas asociaciones entienden que para ser visibles, lucrativas y relevantes deben estar sistematizadas y en la medida de lo posible, digitalizadas. Estas asociaciones son conocedoras también de que la población a la que quieren llegar ya está altamente permeada por lo digital, y por ende son conscientes de la importancia de los estudios antropológicos digitales para entender al usuario y amplificar el alcance del mensaje. Diariamente entidades gubernamentales y privadas buscan integrar a sus equipos talento profesional con experiencia en investigación, producción y emprendimiento digital para colaborar al logro de sus objetivos planteados a corto, mediano y largo plazo.

La demanda de antropólogos digitales es alta para el entendimiento in house; es decir, del cliente interno, la cultura empresarial, las políticas internas y la transformación digital de una empresa. Lo mismo sucede con el entendimiento hacia afuera, es decir, de los usuarios, consumidores y la población; y con las relaciones humanos-máquina, en el encuentro de las poblaciones con las apps, de los tópicos discursivos que se dan en los entornos digitales acerca de temas de actualidad o de interés público.

Un profesional que entiende el alcance y la profundidad de las posibilidades digitales en el entorno humano es un aliado ideal para las instituciones que ven el potencial digital como 
apoyo a sus estrategias de comunicación, publicidad y mercadeo. Los antropólogos digitales proponen diferentes estrategias para la recolección de información, procesamiento de data e identificación de insights relevantes para la toma de decisiones estratégicas de negocio. Además, proponen la triangulación de instrumentos y herramientas como etnografías digitales, netnografía, sociogramas, y escuchas digitales que generan una data cualitativa indispensable a la hora de estudiar al consumidor, es decir, investigación digital.

Hay que entender que en investigación digital el todo está compuesto por el big data y el thick data, siendo el primero la data cuantitativa en mayor escala que explica el qué, y el segundo la data cualitativa que hace parte del entendimiento de los por qué, cómo, para qué, con quién y cuándo. Sin este componente cualitativo es imposible abordar cualquier fenómeno donde intervenga el ser humano. El antropólogo digital trabaja para identificar esa data cualitativa con el input de la data cuantitativa generando estudios más profundos sobre el entendimiento de cualquier fenómeno digital.

El mundo de productos digitales como aplicaciones apps o sitios digitales como webs, blogs, o videojuegos, por nombrar los más consumidos, requieren de la creación e ideación de prototipos que no pueden ser pensados sin centrarse en el usuario. Este enfoque que sitúa al usuario en el centro, requiere de investigación constante en la cadena operativa de estos productos digitales: se hace antes, durante y después e integrando no sólo su percepción ideal de cómo deben ser estos productos digitales, si no también la experiencia de uso, y por último, la reacción al producto final entendiendo su funcionalidad, caducidad y propósito.

Un caso muy sonado que permite ilustrar este tipo de aplicabilidad, es el trabajo de la antropóloga digital Doctora Tricia Wang (2016a, 2016b), precursora del thick data, quien realizó un estudio multidimensional de antropología digital para el gigante Netflix entregando datos cualitativos y contextuales que permitirían a la plataforma entregar un producto enfocado en la personalización de la experiencias; esto se traduce en un producto enfocado en mis gustos personales como usuario y no en los gustos de una población general. La doctora Wang es clara: no es suficiente con invertir en big data, es necesario un insight humano que entienda las tendencias emergentes y las ventajas de escuchar al usuario; Netflix lo hizo y se posicionó. ¿Pero, qué pasa cuando una empresa invierte en big data pero falla en entenderlo y usarlo adecuadamente? El caso de Nokia es una perfecta ilustración de este problema, es una compañía que pasó de ser líder en la fabricación y venta de teléfonos celulares a perder la mayor parte de su negocio, invirtió en big data pero falló en ver el cambio que se estaba gestando en el usuario: el smartphone llegó para quedarse. No sorprende que ahora un gran número de plataformas, apps y videojuegos no salgan al aire sin la intervención de la antropología digital en su concepción, diseño y estrategia de mercadeo, big data e insight humano trabajando juntos.

Entre los espacios de investigación que menos se piensa que pueda intervenir la antropología digital es en estudios de ciencias sociales, dado que la academia aún no involucra 
la antropología digital dentro de las aulas o como propuesta seria de integración al ámbito investigativo. No obstante, los estudios sociales fuera de la academia han volcado su mirada a la antropología digital entendiendo la calidad de data que produce y la información relevante que arroja para los procesos de intervención social. Por ejemplo, una investigación realizada en el año 2014 para la Federación Nacional de Cafeteros de Colombia ${ }^{2}$, arrojó un hallazgo que evidencia la relación de esta población con aspectos digitales, se identificó la relación de los agricultores con medios como YouTube y Facebook, donde esa población se informa acerca de datos relevantes referentes a precios actuales, avances en procesos productivos y fluctuación de productos en los mercados internacionales; además, se forma en mecanismos de control de plagas, optimización de suelos y generación de protocolos de paso de un cultivo a otro. Estos hallazgos han aportado a la creación de contenido desde entes públicos y privados para estas poblaciones y otras similares, con el ánimo de cualificar al caficultor y mejorar así sus condiciones de competitividad, lo que se espera genere una mejora económica para este sector. Esto es un ejemplo de los proyectos de investigación e intervención social, donde la antropología digital puede apoyar y aportar a este entendimiento.

Pensar en la antropología digital fuera de la academia es pensar bidimensionalmente en un mundo que claramente es tridimensional, es un retroceso del estudio del entendimiento humano, es limitar el espacio de acción de futuros antropólogos, es pensar que la humanidad interactúa en los mismos espacios que hace cien años, es negar que la cotidianidad del ser humano sucede tanto en escenarios digitales online como en offline. Es por esto que la academia debe aceptar que la digitalización está sucediendo a nivel mundial, sea que su visión conservadora de la antropología lo quiera o no, y debe prepararse a emprender el estudio de esta nueva normalidad para crear las herramientas conceptuales, teóricas y prácticas para proveer a los antropólogos de las bases necesarias para adaptarse a una realidad laboral que cada vez necesita más de su conocimiento. Es entender que este mundo digitalizado requiere una mirada digital desde la antropología para dar respuestas reales a preguntas actuales.

Otros sectores de las ciencias humanas están haciendo la transición hacia lo digital como potencializador del profesional humano, un caso concreto es la traducción. Con la entrada de la inteligencia artificial que ha potencializado la traducción libre y gratuita en un motor de búsqueda como Google, se pensaría que los traductores humanos son obsoletos, ¿para qué pagar un traductor para que traduzca contenido que se puede traducir gratis? Sin embargo, el insight humano es fundamental para cualquier ejercicio humano y la comunicación es profundamente eso, humana. Sí, en principio los traductores gratuitos funcionan y es posible hacerse una idea

\footnotetext{
${ }^{2}$ Este estudio por ser de carácter privado no ha sido sistematizado para el público, solo para el interesado. El dato que se menciona aquí no fue parte de los objetivos principales de la investigación, no obstante, constituyó un gran hallazgo en término de adopción de tecnologías en estas poblaciones rurales.
} 
general del contenido, pero ninguna compañía posicionada, ningún gobierno, ningún producto líder quiere ver su contenido sometido al escrutinio público por la mediocridad de dicha traducción. Entonces, ¿cómo conjugar el potencial digital con el talento humano en este campo? Es aquí donde entran el software de traducción diseñados para respaldar y facilitar el trabajo del traductor humano, ayudando a la reducción de errores, tiempos de traducción y costos sin reemplazar su experiencia e insight. El deber de la academia en este caso es preparar traductores que, cuando sea necesario, sean capaces de usar y sacar provecho de las herramientas de traducción asistida por computador. Un traductor humano que usa la tecnología digital para optimizar su trabajo.

Involucrar a la antropología digital como parte de la formación de antropólogos, es una manera también de reivindicar una profesión que se pensaba sin éxito en el mundo tecnológico y evidenciar la relevancia que tiene para la humanidad y el futuro de la misma. Llevar la antropología digital a las aulas no es solo un derecho, es también un deber de la antropología con el mundo, y la humanidad. Es repensar lo digital y lo tecnológico como una herramienta creada por el humano para ayudarlo, no para reemplazarlo; es entender que como las revoluciones industriales anteriores, lo digital es una expresión de la capacidad humana para el desarrollo y que merece ser estudiada y entendida como cualquier otra hazaña lograda por el ser humano.

\section{Antropología digital en el mundo laboral colombiano}

Entender la antropología digital como un requerimiento de la sociedad general es una labor que desde distintas instituciones y organizaciones se está llevando a cabo. Para exponer un caso propio en Colombia; la red lab de antropología aplicada de Colombia Antropolab se propuso ahondar en el tema desde la perspectiva laboral, y aunque no es a primera indagación en el país en este tema es una de las más extensas "Encuesta de mercado laborar de antropología en Colombia" que se llevó a cabo en el mes de abril de este año y que arrojó resultados interesantes: por ejemplo de las personas 306 personas participantes cursando pregrado y posgrado en antropología en diversas universidades del país (donde el 63\% son mujeres), sólo el 58\% está empleado actualmente, en las áreas de empleo se destacan proyectos de intervención social $23 \%$, arqueología $17 \%$, docencia universitaria $9 \%$, investigación de mercados $9 \%$, donde antropología digital se presume hace parte de la categoría "otros" con un 14\%. Los entrevistados hablan de los desafíos más grandes entre los que se encuentran: contextos de trabajo con poco tiempo disponible para dedicarse a la investigación, desconocimiento sobre el papel de la

antropología y desconexión entre la formación obtenida en la academia y los requerimientos del mercado de trabajo, además de que la pandemia añadió una preocupación por metodologías digitales que den respuesta a la poca conexión con las comunidades en las condiciones actuales. 
Estos hallazgos nos dan orientaciones acerca de algunas acciones que se pueden desarrollar para que el panorama laboral mejore, por ejemplo: dar a conocer el papel de la antropología digital en el mundo laboral, incluir una cátedra en las instituciones universitarias sobre los escenarios no convencionales de la antropología. Además de esto construir una sistematización de experiencias de los antropólogos digitales y todos aquellos que trabajan en antropología en escenarios no tradicionales. Estas son algunas de las propuestas que aportarían a la generación de mejores condiciones laborales en el área de la antropología.

\section{¿Cómo usar la antropología digital en el mercado de trabajo?}

En lo que atañe al tema de la antropología digital en el mundo laboral es aplicable en todas las áreas; por ejemplo, si se quiere indagar sobre Cómo los medios digitales están moldeando la conducta social, los imaginarios y los discursos de las poblaciones, además de ser una gran herramienta de conexión con las comunidades que, por espacio, geografía y/o accesibilidad no puedan ser abordadas de manera presencial. Hablamos también de que la antropología digital es aplicable en el mercadeo, la investigación en redes sociales y la robótica por mencionar tres de las más comunes. En resumen, la antropología digital tiene cabida en todo escenario que se cuestione sobre la relación humano -tecnología.

Algunas preguntas que podemos hacernos en este sentido son: ¿Cuáles son las herramientas digitales de investigación, intervención y participación que hoy tenemos y cuáles se hacen necesarias en el mundo actual?, ¿Cuál es el alcance de cada una de las herramientas, ¿Cómo se están relacionando nuestras comunidades con el mundo digital?, ¿Cuál es la capacidad de plasmar la vida real y la vida virtual en cada red?, ¿Cuáles son las motivaciones que llevan a nuestras comunidades al uso o desuso de los mecanismos digitales?, ¿Qué mitos existen alrededor del mundo digital hoy en día?. Debemos además entender las redes sociales en términos de los indicadores como like, número de impresiones o enganche. Identificar los códigos digitales como el meme, los gif y el uso que le dan las comunidades a dichos códigos. Estos y otros cuestionamientos nos permitirán acercarnos con mayor claridad a los ejercicios de antropología digital y nos permitirán hallazgos cada vez más cercanos a la realidad de las poblaciones.

La creación de asociaciones, espacios y colectivos donde se alimenten estos debates se hace necesario, pertinente y urgente para que la antropología digital tome fuerza y construya una idea colectiva de aplicabilidad en el mundo real.

Hablando de estos espacios quisiera terminar contando la historia de Antropolab la red lab de antropología aplicada de Colombia. Esta comunidad es producto de una hermosa y grata causalidad o serendipia; Antropolab surge luego de una entrevista que tuve para hablar de la antropología digital, en esta entrevista la pregunta clave fue: ¿Ustedes los antropólogos que se desempeñan en escenarios diferentes al social o académico están organizados?, la respuesta fue 
negativa, es decir, no estábamos organizados y dio pie para publicar un post en Linkedin solicitando que los antropólogos nos uniéramos, intercambiáramos saberes, experiencias, buenas prácticas e hiciéramos tránsito de conocimiento. En resumidas cuentas, la invitación era para organizáramos. La respuesta del público fue reveladora pues más de cuatro mil visualizaciones y más de setenta comentarios me hizo entender que el lugar común de muchos de los antropólogos no sólo de este país si no de muchos otros países, es no tener un lugar para compartir información relevante sobre estos temas y allí es donde entran dos grandes antropólogos que confluyeron para cristalizar la visión de la red: Diana Carolina Henao antropóloga, UX research y Darío Reyes Reina antropólogo de innovación, excelentes profesionales y unas mentes imparables (cf. Reyes, Cruz, Henao 2021). Los tres concretamos la idea de la red y el laboratorio para luego lanzarnos a crear la primera red de antropología aplicada de Colombia que hoy es Antropolab. Antropolab es una comunidad donde el tema principal son los escenarios no convencionales de la antropología como: antropología digital, antropología e innovación, antropología y UX antropología y políticas públicas, y todos los escenarios no tradicionales donde la antropología intervenga. Así nació oficialmente en el mes de mayo del 2020 Antropolab.

Los retos de la red son muchos: en primer lugar, está la financiación para la funcionalidad de la redlab, sin embargo, la pandemia nos permitió nacer como una comunidad digital y desde allí operar $100 \%$, sin necesidad de estar patrocinados por alguna institución, nuestros fondos son propios. En segundo lugar, conformar equipo con diferentes personas es complejo y si a esto le sumamos que crear equipo con compañeros con los que nunca has tenido la oportunidad de interactuar se convierte en un mayor desafío y ese fue nuestro caso, pues Diana, Darío y yo no nos conocíamos antes del post de Linkedin, no obstante, la idea de lo que queríamos de Antropolab fue similar desde el principio y desde esa manera trabajamos para concretar objetivos claros y desarrollar las metas planteadas. En tercer lugar y no menos importante es generar contenido de relevancia para la comunidad; este es uno de los más grandes retos para cualquier comunidad y por esto nuestro primer objetivo fue informar, visibilizar y presentar a los profesionales y las historias que acompañan a estos antropólogos no convencionales. Para esto nos dimos a la tarea de entrevistar a antropólogos en diferentes líneas, para que nos contaran acerca de sus experiencias, así generar tránsito de conocimiento y lanzamos las mentorías. Las mentorías son un programa de 3 meses de duración donde los antropólogos expertos dan su conocimiento a antropólogos en formación sobre distintas líneas de la antropología no convencional. Y hoy en día entendimos que debemos hacer el 2.0 de nuestra red y nos estamos preparando para informar, educar y apoyar con algunas estrategias digitales que verán pronto como los podcast, encuentros sincrónicos, charlas en vivo y unos cursos cortos de conocimiento de antropología y tecnología para empezar. 
Antropolab está ampliando la red de aliados estratégicos como lo son UX COOL Academy que si bien no es un grupo de antropología ha sido un soporte magnífico en la creación de comunidad y en plataforma de streaming, además, hemos apoyado algunas actividades con universidades colombianas como la Fundación Claretiana, Universidad de Antioquia, Universidad Nacional, Universidad ICESI, Universidad Surcolombiana, Universidad del Rosario, Universidad de Santander, la Asociación Nacional de Antropología de Colombia, un grupo de antropología de Perú que es Trasnculturate y estamos en conversaciones con algunas instituciones mexicanas, argentinas y españolas. Esperamos llegar a muchos más países, hoy en día ya tenemos presencia de varios representantes de distintos países con mentores de Colombia, Argentina, México y España.

El futuro de Antropolab es crear nodos propios en diferentes países y desde allí seguir ampliando el impacto de la comunidad y llevando la antropología no convencional a definirse como un área de aplicabilidad total en el mundo actual.

\section{Referências bibliográficas}

MILLER, Daniel. 2011. Tales from Facebook. Cambridge: Polity Press

MILLER, Daniel; SLATER, Dan. 2000. The Internet: An ethnographic Approach. Oxford: Berg. cap 2,6 p 27-55, 145-172

MILLER, Daniel (ed.). 1998. Material culture: Why some things matter. Chicago: University of Chicago Press. Cap 6 P 121-146

PINK, Sarah. 2009. Doing sensory ethnography. Londres: Sage Publications Ltd. p 1-34

PINK, Sarah. 2012. Advances in Visual methodology. Londres: Sage Publications Ltd. cap 7, p 113129

PINK, Sarah, HORST, Heather; POSTILL, John. (2019). Etnografia digital: principios y prácticas. Chile: Ediciones Morata. Cap 1,2,3. P 17-80

REYES, Darío, CRUZ, Tatiana \& HENAO, Diana. 2021. Encuesta mercado laboral de la antropología en Colombia 2021. Disponível em: https://www.academia.edu/48266721/Encuesta_mercado_laboral_de_la_antropolog\%C3\%ADa_en _Colombia_2021 (revisado em 05/2021)

WANG, Tricia. TED. Septembro, 2016a. The human insights missing from big data (Video). Disponível em: https://www.ted.com/talks/tricia_wang_the_human_insights_missing_from_big_data (revisado em 05/2021)

WANG, Tricia. Maio, 2016b. ¿Por qué Big Data necesita Thick Data?, por Tricia Wang. Paz Bernaldo (trad.). Disponível em: https://medium.com/@triciawang/por-qu\%C3\%A9-bigdata-necesita-thick-data-fbbe11dfb088 (revisado em 05/2021) 
sobre la autora

Tatiana Alexandra Cruz

Licenciada en Antropología por la Universidad de Antioquia y especialista en planeamiento urbano por el Colegio Mayor de Antioquia. Actúa como profesora en la Fundación Universidad Clareatiana y hace parte como fundadora del Antropolab.

Autoría: No aplicable

Financiación: No aplicable.

Fecha de recibimiento: $24 / 02 / 2021$

Fecha de aceptación: 02/06/021 